\title{
Can a "good death" be made better?: A preliminary evaluation of a patient-centred quality improvement strategy for severely ill in-patients

\author{
Jeff Powis ${ }^{1}$, Edward Etchells*1, Douglas K Martin ${ }^{2}$, Susan K MacRae ${ }^{2}$ and \\ Peter A Singer ${ }^{2}$
}

Address: ${ }^{1}$ Department of Medicine, University of Toronto, Sunnybrook and Women's College and Health Sciences Centre, 2075 Bayview Avenue, Room C410, Toronto, ON, Canada, M4N 3M5 and '2University of Toronto Joint Centre for Bioethics, 88 College Street, Toronto, ON, Canada, M5G 1L4

Email: Jeff Powis - JeffEPowis@aol.com; Edward Etchells* - edward.etchells@sw.ca; Douglas K Martin - douglas.martin@utoronto.ca; Susan K MacRae - sue.macrae@utoronto.ca; Peter A Singer - peter.singer@utoronto.ca

* Corresponding author

Published: 23 May 2004

BMC Palliative Care 2004, 3:2
Received: 26 January 2004

Accepted: 23 May 2004

This article is available from: http://www.biomedcentral.com/l472-684X/3/2

(c) 2004 Powis et al; licensee BioMed Central Ltd. This is an Open Access article: verbatim copying and redistribution of this article are permitted in all media for any purpose, provided this notice is preserved along with the article's original URL.

\begin{abstract}
Background: Prior studies attempting to improve end-of-life care have focused on specific outcomes deemed important to healthcare providers, with disappointing results. Improvement may be best achieved by identifying concerns important to individual patients, communicating the patients' concerns to the treating medical team, and repeating the process frequently until all concerns are addressed. Our objective was to conduct a preliminary evaluation of this innovative patient-centred quality improvement strategy.

Methods: Initial interviews elicited participants' ideas for improvement, which were then fed back to health care providers by the study investigator. A rapid-cycle change model ensured frequent reassessment and continued feedback. The study involved 36 seriously ill, hospitalized patients on teaching general medical inpatient units of a tertiary care hospital. The main outcome measure was participants' ratings of satisfaction within different domains of care on follow-up interviews.

Results: The proportion of participants who rated various aspects of their care as "excellent" or "very good" on initial interview was $72 \%$ for overall care, $64 \%$ for symptom control, $66 \%$ for level of support, and $75 \%$ for discussions about life sustaining treatments. Patients and families identified many actionable steps for improvement such as; better control of pain and shortness of breath, better access to physicians and medical information, more help with activities of daily living, improving the patient's environment, and shorter waits for nursing care, diagnosis, and treatment. Following feedback to the clinical team, participants reported improvement in overall care (32\%), symptom control (44\%), and support (40\%). Only a minority had further discussions about life sustaining treatments.
\end{abstract}

Conclusion: A patient-centred approach using rapid-cycle change was feasible and shows promise for improving the quality of end-of-life care. It should be evaluated on a larger sample in a controlled trial. 


\section{Background}

Implicit in the question, "What is a good death?" is the question of how to make deaths better. Prior studies of quality improvement in end-of-life care have focused on specific outcomes deemed to be important from the providers' perspective, such as reducing time to first dose of analgesic, educating patients about pain control, or increasing the completion rate of advance directives [1-6]. The largest of these studies, the SUPPORT trial, was unable to demonstrate a significant change in outcome measures dealing primarily with life-sustaining treatment decisions. The focus of the study dealt with physicians' concerns related to end-of-life care. These concerns formed the basis for discussions between study nurses and patients, while patients' concerns were not specifically addressed. In a SUPPORT substudy focused on pain control, a pain intervention was designed, but very few patients received measurable changes in their pain management, and the study was negative [3].

Improvement in end-of-life care may arguably be best grounded in the perspective of individual patients and their families by focusing on issues considered important to them [7-12]. Almost two decades ago Julia Neuberger, former Chief Executive of the King's Fund, met with seriously ill patients in a Boston hospital to identify their concerns and personally fed back these concerns to the medical team and hospital administration (Neuberger J, Personal communication). This strategy involving rapid feedback of individual patients' preferences through direct discussions with the treating medical team has never been formally studied within end-of-life care.

We set out to test the feasibility and potential impact of such a strategy, so we chose a rapid-cycle improvement design involving frequent reassessment and feedback [13]. Our objective was to determine whether this type of patient-centred improvement strategy, with more direct and intense feedback of patient's preferences, might be associated with better patient satisfaction with end of life care. If the results of this preliminary evaluation were encouraging then a larger controlled study would be warranted.

\section{Methods}

\section{Participants and setting}

This prospective study was conducted in January, February, and April 2002 on the general medical inpatient teaching units at Toronto's University Health Network (UHN), Western Site. A Palliative care consult service was involved in patient care if requested by the treating medical team and continued to provide usual care during the study period. Eligible participants were required to have an estimated prognosis of less then one year, and needed to be aware of their diagnosis and estimated prognosis.
The potential participant's prognosis was estimated by the treating medical team. If the participant was unable to respond appropriately to the interviewer (JP) on two separate occasions, a family member or close friend was interviewed instead.

\section{Sampling and sample size}

We approached 63 consecutive potentially eligible participants during the study period. Six did not meet eligibility criteria: one patient's prognosis on further testing was deemed to be greater than one year and five patients were incapable yet did not have family members or close friends to interview. Another 21 refused for the following reasons: too tired (5), too busy dealing with their illness (5), not yet ready to talk about their illness (5), and miscellaneous reasons (6). Therefore, our study sample comprised 36 participants.

\section{Strategies for improvement}

We used information from patients and families to stimulate change. First, we encouraged patients and families to reflect on their experience and needs [14,15]. Second, we identified specific aspects of care that the patients and families felt were high priority for improvement. Third, we relayed this information to the treating senior medical resident and nursing team leader. A medical resident (JP) who had recently worked on the unit relayed the information. No specific advice was given to caregivers on how to best address each participant's concern. For example, in one interview we determined that a patient's eyes were sore; we informed the treating team the patient's eyes were sore, not how to treat the sore eyes. Finally, follow-up interviews sought participants' views about changes in their care, as well as any new suggestions for feedback. Health care providers and participants were very receptive to the feedback process.

\section{Data collection}

One investigator (JP) interviewed all participants. We used a previously developed patient-centred taxonomy of care as a framework for interviews [7]. The interview guide included questions regarding the participants' satisfaction with overall care, symptom control, support from the healthcare team and discussions about life sustaining treatments. Each domain was addressed by both openended questions as well as asking the participant to rate their satisfaction on a scale from "excellent" to "poor", or on follow-up interviews "better", "same", or "worse". Follow-up interviews were conducted approximately every four days and were continued until the patient was discharged, declined further interview, or died. An interpreter was used with non-English speaking participants. All interviews were audiotaped and transcribed. Initial interviews took approximately 30-60 minutes; follow-up 
Table I: Characteristics of participants

\begin{tabular}{|c|c|}
\hline \multicolumn{2}{|c|}{ Baseline Characteristics } \\
\hline Sex & 19 Male, 17 Female \\
\hline Age & Mean 77, Range 49-99 \\
\hline \multicolumn{2}{|c|}{ Interviewee } \\
\hline Patients Only & 12 \\
\hline Patient and Family & 3 \\
\hline Family Only & 21 \\
\hline \multicolumn{2}{|l|}{ Language } \\
\hline Patient's First Language Not English & 19 \\
\hline Translator required for interview & 8 \\
\hline \multicolumn{2}{|l|}{ Resuscitation Status } \\
\hline DNR & 19 \\
\hline Not Addressed at time of initial interview & 12 \\
\hline Addressed yet no DNR order in chart & 2 \\
\hline \multirow[t]{2}{*}{ Specific request } & $\begin{array}{l}\text { To ICU for reversible } \\
\text { causes = I }\end{array}$ \\
\hline & No $A C L S=1$ \\
\hline Full Code & 1 \\
\hline \multicolumn{2}{|l|}{ Primary Diagnosis } \\
\hline Cancer & 16 \\
\hline Cardiorespiratory & 11 \\
\hline Neurological & 6 \\
\hline Hepatic Disease & 3 \\
\hline
\end{tabular}

interviews approximately $15-30$ minutes; and feedback to caregivers took approximately 15-30 minutes.

\section{Analysis of data}

We listed the concerns identified by the patients and families at initial interview, then grouped these concerns by theme, using content analysis [16]. We summarized the number of patients rating their care as improved, unchanged, or worse after each cycle of interviews and feedback. The main measure of improvement was patient or family ratings of care.

\section{Research ethics}

This study was approved by the UHN Committee for Research on Human Subjects. All participants provided written informed consent.

\section{Results}

The baseline demographics of the 36 participants are shown in Table 1.

Two participants withdrew because they were too tired to continue with the questioning. Of the remaining 34, 13 patients died in hospital, 20 patients were discharged, and one was alive in hospital at the end of the study period. After the initial interview 6 patients were discharged and 3 died, leaving 25 participants who underwent the first follow-up interview. Eleven patients were discharged and

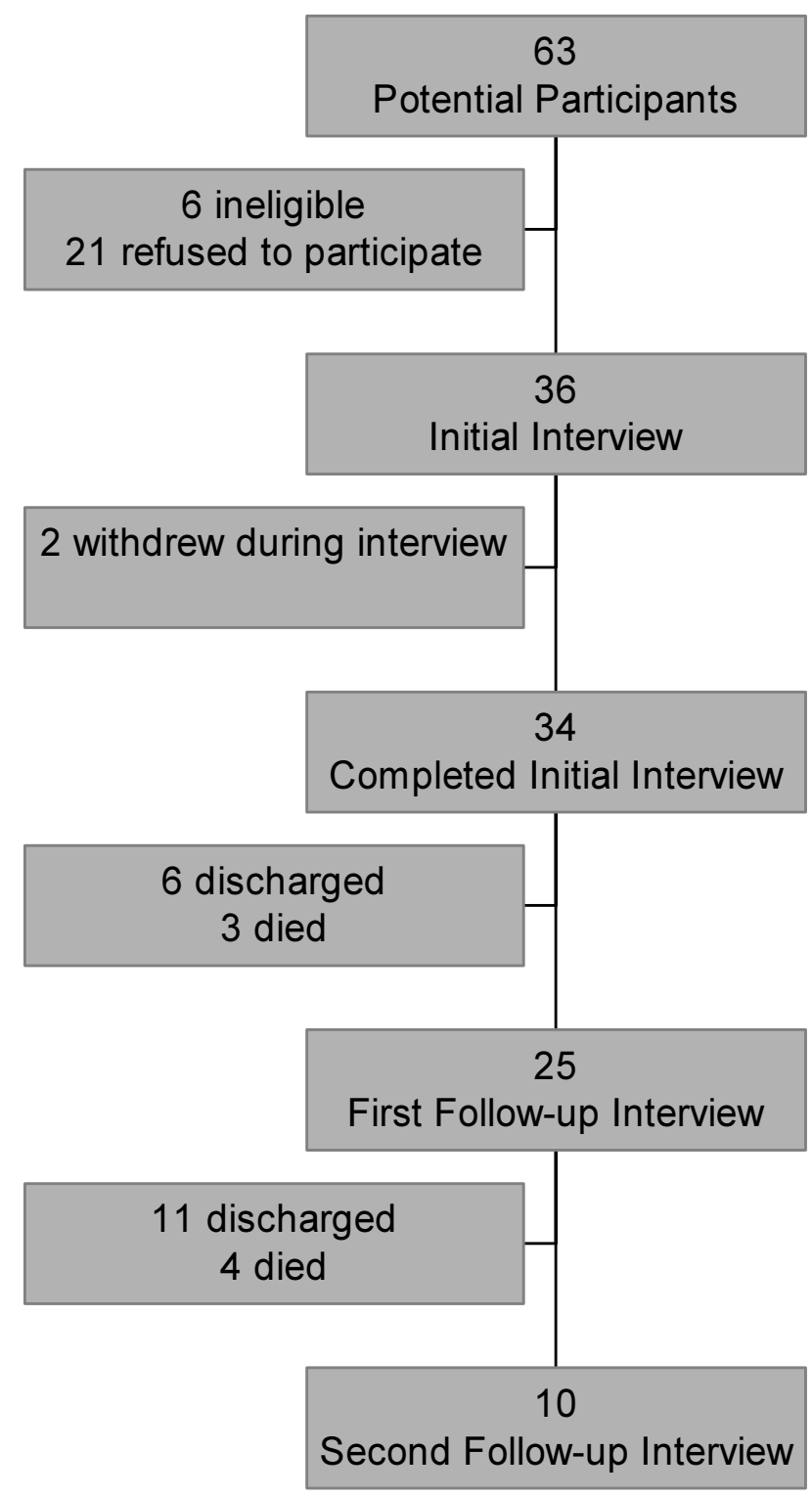

Figure I

Patient follow-up during study period

4 died after the first follow-up interview, leaving 10 participants for the second follow-up interview (See Figure 1).

\section{Initial interview}

The average number of days from admission to initial interview was 10. Patients were interviewed alone 33\% (12 of 36) of the time, families were interviewed alone $58 \%$ (21 of 36 ) and patients and families were interviewed together $8 \%$ ( 3 of 36) of the time. Satisfaction with end-of-life care on the initial interview is shown in Figure 2. The proportion of participants who rated various 


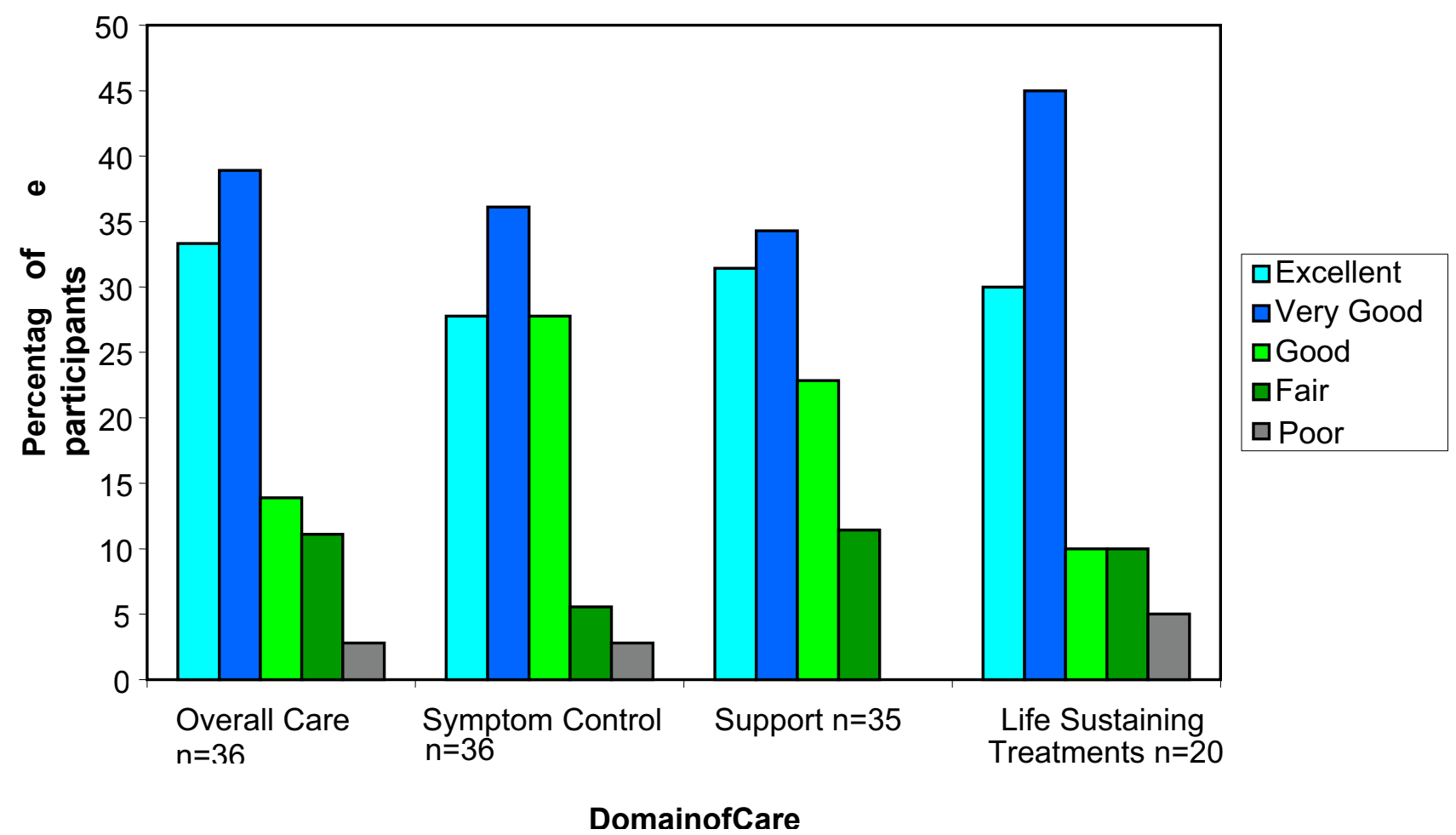

Figure 2

Participants' ratings of satisfaction with care at time of initial interview

aspects of their care as "excellent" or "very good" was $72 \%$ for overall care, $64 \%$ for symptom control, and $66 \%$ for level of support. The median response for all domains was "very good". 20 (57\%) of the participants reported discussions with their treating team about what treatments they might want if they were to get sicker. Of these, 75\% rated their satisfaction with these discussions as "excellent" or "very good". The median response was "very good". Of the 16 individuals who did not have discussions about life sustaining treatment issues at the time of initial interview only 2 requested further discussions as a point for feedback.

The initial concerns or issues raised by the participants and relayed to the health care team are grouped according to theme as shown in Table 2.

\section{First follow-up interview}

The follow-up data for overall care, symptom control and support are shown in Figure 3. Following feedback to the clinical team, participants reported improvements in overall care (32\%), symptom control (44\%), and support $(40 \%)$. One participant rated their initial satisfaction with symptom control as being excellent, yet at follow-up interview felt it was worse because of the new onset of painful oral ulcers.

Of the 25 participants who had at least one follow-up interview, only six reported subsequent discussions about life sustaining treatments. Five of the six rated their satisfaction with these discussions as the same, and one rated their satisfaction as better. Four of the six had identified the need for further discussions at the time of the initial interview.

\section{Second follow-up interview}

As shown in Figure 4, the responses from the second follow-up interview show less improvement but the trend continues for overall care with $30 \%$ of this small sample saying their care had improved, and only $10 \%$ saying it had worsened. There was no continued improvement for symptom control and support. Only three participants reported subsequent discussions about life sustaining treatment at second follow-up. Two of the three rated their satisfaction with discussions as the 'same', and one rated their satisfaction as better. Two of the three had 
Table 2: Participant priorities for improving care

\begin{tabular}{|c|c|}
\hline Issue & Frequency \\
\hline Alleviate Symptoms & 34 \\
\hline Pain & 9 \\
\hline Shortness of breath & 7 \\
\hline Thirst & 3 \\
\hline Other symptoms (gastrointestinal, cough, agitation oversedation, headache, feeling cold, dry skin, itching) & 15 \\
\hline Reduce Delays & 28 \\
\hline Delays in daily bedside care & 9 \\
\hline Delays in diagnosis or treatment & 8 \\
\hline Delay in transfer from emergency room to ward & 6 \\
\hline Delays in discharge to home & 5 \\
\hline Improve Daily Care & 29 \\
\hline Assist with activities of daily living & 9 \\
\hline Improve hospital environment & 6 \\
\hline Arrange help at home & 4 \\
\hline Personalize medical treatment & 3 \\
\hline Improve food & 3 \\
\hline Ensure staff are aware of special care needs & 2 \\
\hline Coordinate timing of diagnostic tests & I \\
\hline Obtain palliative care consultation & 1 \\
\hline Better Access to MDs/Medical information & I I \\
\hline Improve Therapeutic Alliance & 10 \\
\hline Identify responsible physician & 3 \\
\hline Avoid unprofessional behaviors and comments & 3 \\
\hline Reduce changes in nursing staff & 2 \\
\hline Avoid repetitive questioning & I \\
\hline Apologize for medical error & I \\
\hline Address Emotions & 9 \\
\hline Fear of abandonment & 7 \\
\hline Loneliness & 2 \\
\hline Discuss Life Sustaining Treatments & 8 \\
\hline Further discussions & 5 \\
\hline Prior discussion should have been done differently & 3 \\
\hline Provide Personal Support & 3 \\
\hline Write letter so family can visit from outside country & I \\
\hline Wants to see chaplain & 1 \\
\hline Want someone to speak to my loved one in their native language about their medical condition & I \\
\hline
\end{tabular}

identified the need for further discussions at the previous interviews.

\section{Discussion}

To our knowledge, this is the first evaluated attempt in a clinical setting to improve end-of-life care by combining rapid-cycle change and a patient-centred perspective. In our patient population we found plenty of room for improvement with $28 \%$ of patients and families reporting their satisfaction with overall care as only "good", "fair", or "poor." Patients and families identified actionable steps for improvement such as, better control of pain and shortness of breath, better access to physicians and medical information, more help with activities of daily living, improvement of the patient's environment and shorter waits for nursing care, diagnosis, and treatment. Our strategy was associated with improvements in ratings of over- all care, symptom control, and support. We made little improvement in the area of discussions regarding life sustaining treatments as few patients asked for further discussions on life sustaining treatment issues and such discussions rarely occurred without feedback.

The reported improvement in multiple domains of endof-life care in our study is in contrast to the disappointing results of previous trials to improve end-of-life care $[3,4]$. The largest of these studies, the SUPPORT trial failed to change physicians' behaviors or measurable outcomes [4]. One possible reason for the SUPPORT trials' lack of significant change was the untargeted focus on life-sustaining treatments or pain. By contrast, we focused on issues deemed important by individual patients, and found pent up concerns of dying patients and their families that had not been addressed by the standard provider- 


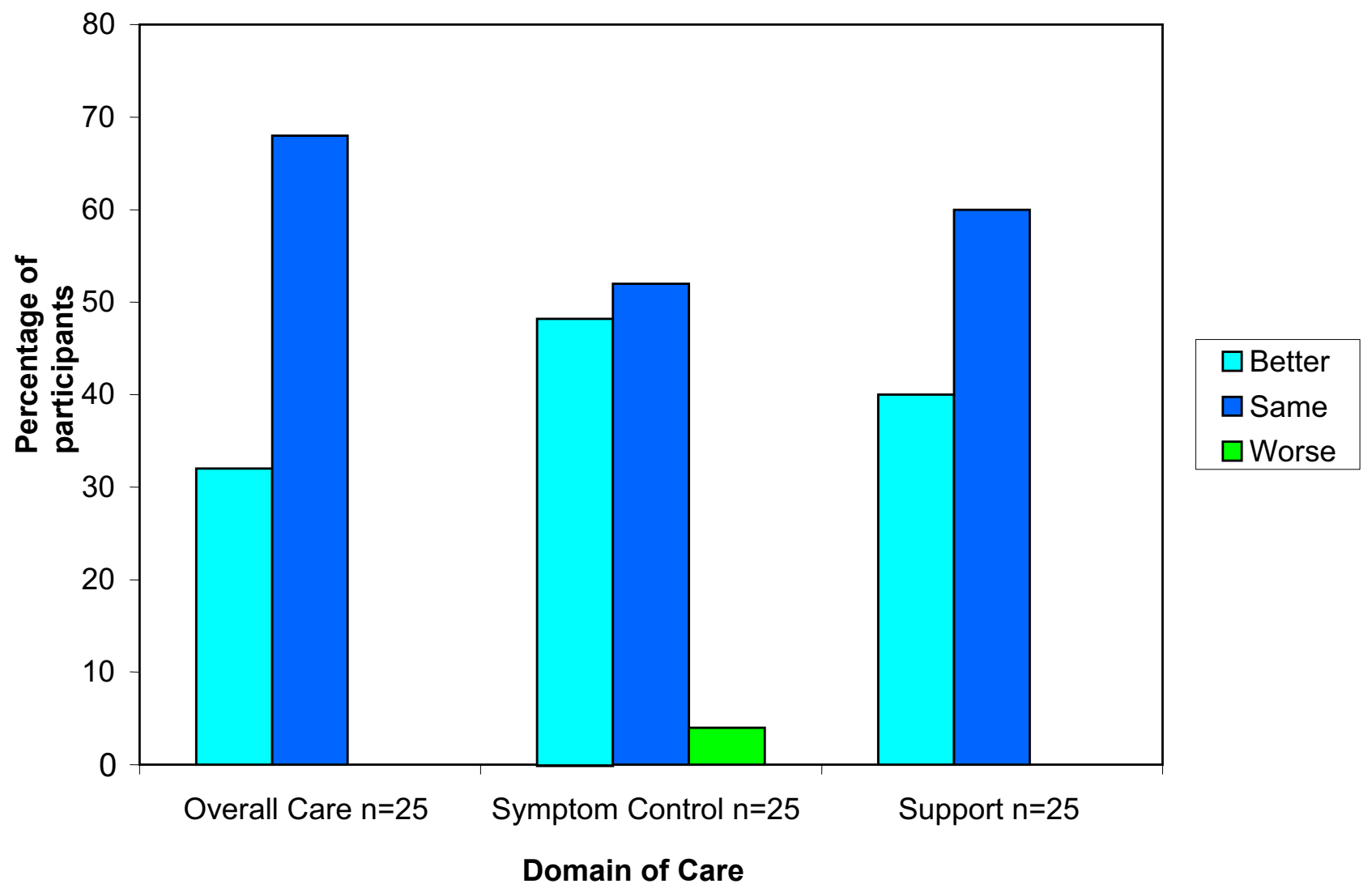

Figure 3

Participants' evaluation of the change in care at time of follow-up interview one

focused approach to end-of-life care. Few patients identified life sustaining treatment discussions as a priority, and for many of our patients, pain was not the primary symptom of concern. Our approach released patients' concerns into the care process allowing for individually important change to occur. We strived for frequent and complete feedback to our treating physicians. In the SUPPORT study, although complete feedback was the intent, only $34 \%$ of study physicians recalled receiving any feedback [3].

The rapid-cycle change model utilized in this study has had prior successes in end-of-life care. In 1997 the Institute for Healthcare Improvement and the Centre to Improve Care of the Dying used a rapid cycle change program to improve important processes of care, such as time from request to first dose of analgesia $[1,2]$. The flexibility of the rapid cycle change method may be well suited for the complexities of end of life care.
There are several limitations to this study. First, there is no concurrent control group so we do not know what participants' perceptions would have been in the absence of any intervention. In our attempt to initiate change we tested this innovative approach for feasibility and utility. It would have been premature to do a controlled trial, but now that we have defined a clear intervention it would be reasonable to proceed with such a study. Second, our data reflects the experiences of a small group of patients at a single hospital. However, despite the relatively small sample size, we were able to show that our innovative approach led to improvement. Third, the intervention and satisfaction assessments were performed by a single resident physician (JP) known to the healthcare staff. The positive effect of the intervention may reflect the popularity and enthusiasm of the interviewer. However, the interviewer (JP) used a standardized questionnaire to identify participant's concerns and acted simply as a conduit between participants and their caregivers. The 


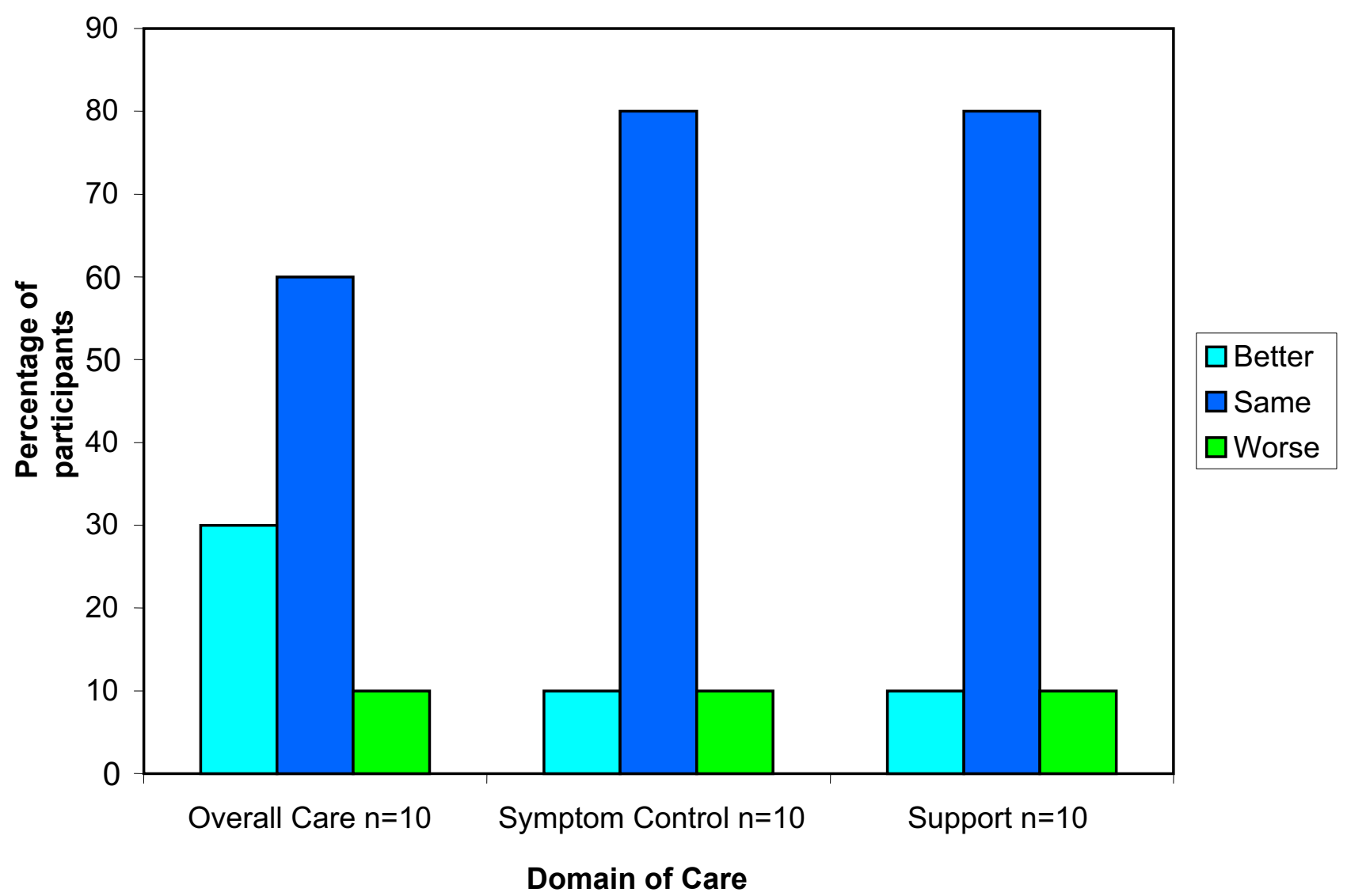

Figure 4

Participants' evaluation of the change in care at time of follow-up interview two

changes in care were carried out by many different members of the healthcare team. We believe that the major impact was the feedback of patients' concerns, and the role of the individual interviewer was secondary. Finally, there were a relatively large number of potential participants $(33.3 \%)$ who refused to participate. The number of refusals did not surprise us, as it is consistent with prior work with severely ill patients [1,2]. Many patients refused because they were too tired, too busy dealing with illness, or not read to talk about their illness. We suspect that these potential participants were not completely satisfied with their care, and would also have responded to a patient-centred quality improvement strategy

Our study tested the utility of an innovative initiative to induce change within end-of-life care. We found that our patient-centred approach using rapid cycle change was feasable and shows promise for improving the quality of end-of-life care. It should be evaluated on a larger sample in a controlled trial

\section{Authors' contributions}

JP interviewed all participants and communicated feedback to the treating medical teams. All investigators were involved in development of the quality improvement strategy, in data analysis and manuscript preparation.

\section{Competing interests}

None declared.

\section{Acknowledgements}

DKM is supported by an Ontario Ministry of Health and Long-Term Care Career Scientist award. PAS is supported by a Canadian Institutes of Health Research Distinguished Investigator award. This research project was support by The University Health Network and the University of Toronto Joint Centre for Bioethics. We would like to thank the staff on 8A, 8B and $3 \mathrm{~A}$ Fell for their assistance in implementing participants' suggestions for improvement. 


\section{References}

I. Lynn J, Schall M, Milne C, Nolan K, Kabcenell A: Quality in end of life care: insights from two collaboratives. Jt Comm J Qual Improv 2000, 26:254-267.

2. Lynn J, Nolan K, Kabcenell A, Weissman D, Milne C, Berwick DM: Reforming care for persons near the end of life: the promise of quality improvement. Ann Intern Med 2002, I 37: I I 7-I 22.

3. Desbiens NA, Wu AW, Yasui Y, Lynn J, Alzola C, Wenger NS, Connors A.F.,Jr., Phillips RS, Fulkerson W: Patient empowerment and feedback did not decrease pain in seriously ill hospitalized adults. Pain 1998, 75:237-246.

4. A controlled trial to improve care for seriously ill hospitalized patients. The study to understand prognoses and preferences for outcomes and risks of treatments (SUPPORT). The SUPPORT Principal Investigators. JAMA I995, 274: I59|-I598.

5. Danis M, Southerland LI, Garrett JM, Smith JL, Hielema F, Pickard CG, Egner DM, Patrick DL: A prospective study of advance directives for life-sustaining care. N Engl J Med |99|, 324:882-888.

6. Schneiderman LJ, Kronick R, Kaplan RM, Anderson JP, Langer RD: Effects of offering advance directives on medical treatments and costs. Ann Intern Med 1992, I I 7:599-606.

7. Singer PA, Martin DK, Kelner M: Quality end-of-life care: patients' perspectives. JAMA 1999, 28I:I63-I68.

8. Steinhauser KE, Christakis NA, Clipp EC, McNeilly M, Mclntyre L, Tulsky JA: Factors considered important at the end of life by patients, family, physicians, and other care providers. JAMA 2000, 284:2476-2482.

9. Cleary PD, Edgman-Levitan S: Health care quality. Incorporating consumer perspectives. JAMA 1997, 278: |608-16I2.

10. Wensing $M$, Elwyn G: Methods for incorporating patients' views in health care. BMJ 2003, 326:877-879.

I I. Good care of the dying patient. Council on Scientific Affairs, American Medical Association. JAMA 1996, 275:474-478.

12. Hanson LC, Danis M, Garrett J: What is wrong with end-of-life care? Opinions of bereaved family members. J Am Geriatr Soc 1997, 45: I339-1344.

13. Berwick DM: A primer on leading the improvement of systems. $B M J$ 1996, 3 1 2:619-622.

14. Sepucha KR, Belkora JK, Mutchnick S, Esserman LJ: Consultation planning to help breast cancer patients prepare for medical consultations: effect on communication and satisfaction for patients and physicians. J Clin Oncol 2002, 20:2695-2700.

15. Greenfield S, Kaplan S, Ware J.E.,Jr.: Expanding patient involvement in care. Effects on patient outcomes. Ann Intern Med 1985, 1 02:520-528.

16. Manning PK, Cullum-Swan B: Narrative, content and semiotic analysis. Handbook of Qualitative Research Edited by: DenzinNK and LincolnYS. Thousand Oaks, Sage Publications Inc.; 1994:463-477.

\section{Pre-publication history}

The pre-publication history for this paper can be accessed here:

http://www.biomedcentral.com/1472-684X/3/2/prepub 